
OPTIMIZATION OF MULTIMAGNETOMETER SYSTEMS

ON A SPACECRAFT

\author{
F.M. Neubauer* \\ Laboratory for Extraterrestrial Physics \\ Goddard Space Flight Center \\ Greenbelt, Maryland
}

*NAS-NRC Postdoctoral Resident Research Associate 
ABSTRACTi

The accuracy of a magnetic field experiment on a spacecraft is sometimes limited by the spacecraft field and not by the accuracy of the magnetometer itself. It has recently been suggested by Ness and collaborrators that $N \geq 2$ magnetometers on a radial boom may be employed to determine the first N-1 multipole contributions to the spacecraft field and thereby improve the total accuracy of a magnetic field experiment. In this study the total error for systems of one to four magnetometers is investigated. The optimal magnetometer locations, for which the total error is a minimum, are found for given boom-length, instrument errors and magnetic field models characteristic for spacecraft with only a restricted or ineffective magnetic cleanliness program 1ike Mariner-Venus-Mercury 1973. It is found that the error contribution by the magnetometer inaccuracy is increased as the number of magnetometers is increased whereas the spacecraft field uncertainty is diminished by an appreciably larger amount. Therefore, for the field models chosen and a boom-length less than $10 \mathrm{~m}$ from the spacecraft center there is always a gain in accuracy compared with one magnetometer. For example, for a boom-length of $6 \mathrm{~m}$, instrument errors of $0.1 Y$ and a rather conservative model describing MVM73 the total error bounds are $2.1 \gamma, 0.856 \gamma, 0.822 \gamma$ and $0.807 \gamma$ for one to four magnetometers respectively. A less conservative model yields $2.1 \gamma, 0.486 \gamma$ 
$0.379 \gamma$ and $0.355 \gamma$, respectively. Thus the results of this investigation support the conclusion that the multimagnetometer method is especially advantageous for observing geoastrophysical fields which are of the same order or smaller than the spacecraft fields at the end of a boom of technically reasonable length. 
The accuracy of a magnetic field experiment on a spacecraft is sometimes limited by the spacecraft field and not by the accuracy of the magnetometer itself. This problem has been discussed recently in two papers by Ness and collaborators [Ness, 1970; Ness et a1. 1971]. A recently suggësted method to partly overcome this difficulty is to use $N \geq 2$ magnetometers on a radial boom which yields an estimate of the first $N-1$ multipole contributions of the spacecraft field and the ambient field and thereby improve the accuracy of the experiment [Ness et al., 1971]. A dual triaxial magnetometer system is being planned to be flown on the Mariner-Venus-Mercury 1973 mission (MVM73).

It is the purpose of this paper to present an investigation of the important problem of optimizing the position of the magnetometers along a boom of given length to yield a minimized total error. We shall restrict ourselves to at most four magnetometers, which seems to be a practical limit due to weight, power and financial considerations. In section 1 the error analysis is presented, in section 2 the analysis is applied to some illustrative cases and the overall results are discussed in section 3 . 


\section{Error Analysis}

We assume that a radial magnetometer boom is given, which defines a line on which we choose the origin of our coordinate system $0^{\circ}$. If all magnetic sources of significance are inside a sphere of radius a around the origin, the spacecraft field on the boom can be represented [Ness et al., 1971] exactly by

$$
\mathrm{B}_{\mathrm{sc}, \mathrm{i}}=\sum_{\ell=1}^{\infty} \overline{\mathrm{B}}_{\ell \mathrm{i}}\left(\frac{\mathrm{r}_{1}}{\mathrm{r}}\right)^{2+\ell} \quad \text { for } \mathrm{r} \geq \mathrm{a},
$$

where $i=1,2,3$ denote the $x, y, z$-components in a suitably chosen frame of reference. Note, that we have chosen the $\bar{B}_{\ell i}$ to be the multipole fields at $r=r_{1}$, the outer tip of the boom measured from the origin in contrast to the usage by Ness et al. [1971]. This choice makes the formalism more compact. As already noted by Ness et al. [1971] one can see from equation 1 , that it is advantageous to place the $N$ magnetometers on one boom so that each multipole contribution is characterised by only three vector components instead of $2 l+1$ quantities as in the case of arbitrary locations, where $l$ denotes the multipole order. For $N>2$ this has the consequence that with a given number of magnetometers higher multipole contributions can be removed from the total error. In addition one magnetometer boom is technically superior to several booms. The bars on $\bar{B}_{a m, i}$ and $\bar{B}_{l_{i}}$ denote that these are the exact physical quantities without error. At a distance $r$, the total 
field vector which includes the homogeneous ambient field to be measured, is given then by

$$
\mathrm{B}_{i}=\overline{\mathrm{B}}_{\mathrm{am}, \mathrm{i}}+\sum_{\ell=1}^{\infty} \overline{\mathrm{B}}_{\ell \mathrm{i}}\left(\frac{\mathrm{r}_{1}}{\mathrm{r}}\right)^{2+\ell}
$$

A magnetometer at position $r$ would then have the following reading

$$
\mathrm{B}_{\mathrm{obs}, \mathrm{i}}=\mathrm{B}_{z 0, i}+\overline{\mathrm{B}}_{\mathrm{am}, \mathrm{i}}+\sum_{\ell=1}^{\infty} \overline{\mathrm{B}}_{\ell \mathrm{i}}\left(\frac{\mathrm{r}_{1}}{\mathrm{r}}\right)^{2+\ell}
$$

where $\mathrm{B}_{\mathrm{zo}, i}$ is the instrument error of the magnetometer reading due to zero-offset, noise and quantization uncertainty. If we assume $N$ magnetometers for each component, on the boom at positions $r_{i, k}(k=1, \cdots N)$, we obtain $N$ equations for each value of $i=1,2,3$.

$$
\mathrm{B}_{\mathrm{obs}, i, k}=\mathrm{B}_{z 0, i, k}+\bar{B}_{\mathrm{am}, i}+\sum_{\ell=1}^{\infty} \bar{B}_{\ell i}\left(\frac{r_{1}}{r_{i k}}\right)^{2+\ell}
$$

Without loss of generality we can choose the $r_{i, k}$ according to the following inequalities:

$$
r_{1}=r_{i, 1}=r_{i, 2}>r_{i, 3} \cdots>r_{i, N} \geq a \quad i=1,2,3
$$

It should be noted that in our formulation the positions of the mono axial magnetometers for a given $k$ could be different for the three components and indeed all $3 \mathrm{~N}$ monoaxial magnetometers may have no two radial distances the same. For all cases of this 
study $r_{i, 1}$ is taken to be $r_{1}$. We now try to approximate the spacecraft field by the first $N-1$ multipoles.

If the magnetometers were ideal detectors, i.e., without error, we would have the following $N$ equations to solve for the ambient field for each i:

$$
\mathrm{B}_{\mathrm{obs}, \mathrm{i}, \mathrm{k}}=\mathrm{B}_{\mathrm{am}, \mathrm{i}}+\sum_{\ell=1}^{\mathrm{N}-1} \mathrm{~B}_{\ell \mathrm{i}}\left(\frac{\mathrm{r}_{1}}{\mathrm{r}_{\mathrm{i}, \mathrm{k}}}\right)^{\ell+2} \mathrm{k}=1, \ldots \mathrm{N}
$$

The unbarred quantities $B_{a m, i}$ and $B_{\ell i}$ can be considered as estimates of the ambient field and the first $N-1$ multipole fields, respectively. This set of linear equations can be solved for $B_{a m, i}$ It is convenient to introduce the quantities $\rho_{i, k}=\frac{r_{1}}{r_{i, k}}$ :

$$
B_{a m, i}=\frac{1}{\operatorname{det}\left(\rho_{i}\right)}\left|\begin{array}{lllll}
B_{o b s, i, 1} & 1 & 1 & \cdots & 1 \\
B_{o b s, i, 2} & \rho_{i, 2}^{3} & \rho_{i, 2}^{4} & \cdots & \rho_{i, 2}^{N+1} \\
B_{o b s, i, 3} & \rho_{i, 3}^{3} & \vdots & \cdots & \vdots \\
\vdots & \vdots & \vdots & & \vdots \\
B_{o b s, i, N} & & & & \rho_{i, N}^{N+1}
\end{array}\right|
$$

with

$$
\operatorname{det}\left(\rho_{i}\right)=\left|\begin{array}{ccccc}
1 & 1 & 1 & \ldots & 1 \\
1 & 3 & \vdots & \ldots & \rho_{i, 2}^{N+1} \\
1 & \rho_{i, 2}^{3} & \vdots & & \vdots \\
1 & \rho_{i, 3}^{3} & \vdots & & \vdots \\
\vdots & \vdots & \vdots & & \vdots \\
1 & \rho_{i, N}^{3} & & \cdots & \rho_{i, N}^{N+1}
\end{array}\right|
$$


Here $\rho_{i}$ stands symbolically for the set $\rho_{i, 1}, \rho_{i, 2} \cdots \rho_{i, N} \cdot$ The determinants can be expanded with respect to the first column

$$
\operatorname{det}=\sum_{k=1}^{N}(-1)^{k+1} \text { sub-det }\left(k ; \rho_{i}\right)
$$

For sub-det $\left(k ; \rho_{i}\right)$ a relatively simple expression can be found by using some general algebraic theorems:

$$
\operatorname{sub-det}\left(k ; \rho_{i}\right)=\prod_{\substack{n=2 \\ n \neq k}}^{N} \rho_{i, n}^{3} \prod_{\substack{m=1 \\ m \neq k}}^{n-1}\left(\rho_{i, n}-\rho_{i, m}\right)>0
$$

The inequality is fulfilled if inequalities $4 \mathrm{~b}$ are satisfied. For $\mathrm{n}=\mathrm{k}$ or $\mathrm{m}=\mathrm{k}$ a factor one has to be set in the products defining sub-det $\left(k ; \rho_{i}\right)$ in equation $6 \mathrm{~d}$. $B_{a m, i}$ is given by

$$
\begin{aligned}
B_{a m, i} & =\sum_{k=1}^{N}(-1)^{k+1} \frac{\text { sub-det }\left(\rho_{i} ; k\right)}{\operatorname{det}\left(p_{i}\right)} B_{o b s, i, k} \\
& \equiv \sum_{k=1}^{N} \beta_{i k}\left(\rho_{i}\right) B_{o b s, i, k}
\end{aligned}
$$

where $6 \mathrm{~d}$ also defines the matrix $\beta_{i k} \cdot$ We are interested here in the error in the estimate $B_{a m, i}$, i.e., $\Delta B_{i}=B_{a m, i}-\bar{B}_{a m, i}$ Using equation 4 and some general properties of determinants we obtain: 


$$
\begin{aligned}
\Delta B_{i} & =\sum_{k=1}^{N} \beta_{i k}\left(\rho_{i}\right) \cdot B_{z o, i, k} \\
& +\sum_{k=1}^{N} \beta_{i k}\left(\rho_{i}\right) \cdot B_{R N, i, k}
\end{aligned}
$$

where

$$
\mathrm{B}_{\mathrm{RN}, \mathbf{i}, \mathbf{k}}=\sum_{\ell=\mathrm{N}}^{\infty} \overline{\mathrm{B}}_{\ell \mathbf{i}} \rho_{\mathbf{i}, \mathbf{k}}^{2+l}
$$

This represents the exact equation for the total error. The first part represents the error due to the inaccuracy of each single magnetometer and the second part the error due to the residual spacecraft fields $B_{R N, i, k}$ determined by the multipoles of orders $2^{N}$ to infinity. An important point can be noted from equation 7. The error $\Delta \mathrm{B}_{i}$ in the ambient field components is completely independent both of the ambient field $\bar{B}_{a m, i}$ and of the first multipole fields $\bar{B}_{\ell i}$, with $\ell=1, \ldots N-1$.

A rigorous error analysis would use the statistical

properties of $B_{z O, i, k}$ and $B_{R N, i, k}$ to derive first an expected value for $\Delta B_{i}$. Since the error statistics of $B_{z o, i, k}$, containing noise errors, digitization errors and zero-offset errors, is relatively well known to be symmetric around $B_{z o, i, k}=0$, we obtain:

$$
\left\langle\Delta \mathrm{B}_{i}\right\rangle=\sum_{\mathrm{k}=1}^{N} \beta_{i k}\left(\rho_{i}\right)<\mathrm{B}_{\mathrm{RN}, \mathrm{i}, \mathrm{k}}>\text {, }
$$


where $\left\langle\Delta \mathrm{B}_{i}>\right.$ could be referred to as systematic error. For $\mathrm{B}_{\mathrm{RN}, \dot{i}, \mathrm{k}}>$ we could for example take the values from the last mapping of the spacecraft field before launch. After deriving $<\Delta B_{i}>$ the expected value of $\bar{B}_{a m, i}$ would be

$$
\overline{\mathrm{B}}_{\mathrm{am}, i}>=\mathrm{B}_{\mathrm{am}, i}-<\Delta \mathrm{B}_{i}>
$$

We would then define the statistical error as the value of $\left(\sum_{i=1}^{3}\left(\Delta \mathrm{B}_{i}-\left\langle\Delta \mathrm{B}_{i}>\right)^{2}\right)^{\frac{1}{2}}=\delta \mathrm{B}\right.$ which with a very low probability (for example $10^{-6}$ ) will be exceeded statistically. Such an approach is not possible at the present time, since the statistics of unpredictable changes of spacecraft fields is generally only very poorly known.

We use, therefore, a much simpler approach. First, we note that the equations for the single components in equation 7 are not coupled. We can therefore restrict ourselves to one component and henceforth drop the subscript $i$ denoting the components. The problem of optimization can then be stated as follows: Let all the magnetic sources be included in a sphere of radius a and let a boom of length $r_{1}$ from the center of the sphere be given. Furthermore let the uncertainty of a magnetometer reading $\left|\mathrm{B}_{\mathrm{zo}, \mathrm{k}}\right| \leq \mathrm{B}_{\mathrm{M}, \mathrm{k}}>0$ be given as well as a worst case magnetic field distribution along the boom. Then the question is: For what distances of the magnetometers from 
the origin $r_{k}(k=1,2, \ldots N)$ is the error in a given component least? We shall consider the cases $N=1,2,3,4$. From equation 7 we obtain

$$
\begin{aligned}
& \Delta B<\Delta B_{\max }=\frac{1}{\mid \operatorname{det}(\rho)} \mid\left\{\sum_{k=1}^{N} \operatorname{sub-det}(\rho ; k)\left|B_{z 0 ; k}\right|\right. \\
& \left.+\mid \sum_{\mathrm{k}=1}^{\mathrm{N}}(-1)^{\mathrm{k}+1} \text { sub-det }(\rho ; \mathrm{k}) \mathrm{B}_{\mathrm{RN}, \mathrm{k}} \mid\right\}
\end{aligned}
$$

or

$$
\begin{aligned}
\Delta B_{\max } & =\left|\frac{1}{\operatorname{det}(\rho)}\right|\left\{\sum_{k=1}^{N} \operatorname{sub-\operatorname {det}}(\rho ; k) \cdot B_{M, k}\right. \\
& \left.+\mid \sum_{k=1}^{N}(-1)^{k+1} \text { sub-det }(\rho ; k) B_{R N, k} \mid\right\}
\end{aligned}
$$

$\Delta \mathrm{B}_{\max }$ can be considered as an upper bound on the error $\Delta \mathrm{B}$.

2. Results of Optimization of Upper Bound on Error $\Delta \mathrm{B}_{\max }$ for Various Parameters and Field Models

In this section we apply equation 11 for several spacecraft field models and geometrical configurations. Before doing this two points may be noted. Because of the remark after equation 7 the quantities $B_{R N, k}$ giving the spacecraft field at $r=r_{k}$ without the first $N-1$ multipole contributions could be replaced by the complete magnetic field components $\mathrm{B}_{\mathrm{sc}, \mathrm{k}}$ at $\mathbf{r}=\mathrm{r}_{\mathrm{k}}$. Secondly the following "similarity laws" hold: 


$$
\Delta B_{\max }=B_{M, 1}: f\left(\frac{r_{1}}{a} ; N ; \frac{B_{M, 2}}{B_{M, 1}} \cdots \frac{B_{M, N}}{B_{M, 1}} ; \frac{B_{S c}}{B_{M, 1}} \cdot\left(\frac{r}{r_{1}}\right)\right)
$$

Thus, if the accuracy of all magnetometers is improved by a factor of $q$ and the value of the spacecraft field is decreased by a factor $q$ everywhere, the optimal magnetometer positions do not change, and $\Delta \mathrm{B}_{\max }$ is reduced by a factor of q,etc.

We shall present results for the following choices of our parameters:

$$
\begin{aligned}
& a=1 \mathrm{~m} \\
& 4 \mathrm{~m} \leq \mathrm{r}_{1} \leq 10 \mathrm{~m} \\
& \mathrm{~B}_{\mathrm{M}, \mathrm{k}}=0.1 \mathrm{Y} \text { for all k}
\end{aligned}
$$

The choice $a=1 \mathrm{~m}$ for the sphere around the sources corresponds approximately to the main body of a Mariner-type spacecraft (without appendages) or an OGO-type spacecraft, whereás application to the smaller IMP- and Pioneer-type spacecraft would be possible using the similarity results. The origin $O^{\prime}$ will then be close to the geometrical spacecraft center.

The value of $0.1 \gamma$ for the absolute accuracy of a single magnetometer is achievable by present day flux-gate magnetometers, with flipper devices for example, for a bandwidth of $1 \mathrm{~Hz}$ and a digitization window of $0.02 \%$. The following field models are used: 


$$
\mathrm{B}_{\mathrm{SC}}(\mathrm{r})=\mathrm{B}_{\text {dipole }}\left(\frac{\mathrm{a}}{\mathrm{r}}\right)^{3} \sum_{l=0}^{4}\left(\mathrm{f} \cdot \frac{\mathrm{a}}{\mathrm{r}}\right)^{\ell}
$$

with the values of $B_{\text {dipole }}$ and $f$ given in Table 1 .

A11 magnetic models contain multipole contributions up to the 32-pole. The models of odd number have equal multipole contributions at $r=a$ [see for example Ness et al, 1971], whereas the even numbers describe models with equal multipole fields at $r=2 a$, an even more conservative estimate of the nature of the spacecraft field. The success of the multimagnetometer method depends on the relative size of the field components $B_{R N, i, k}$ compared with the total spacecraft field components ${ }^{B}{ }_{S C, i, k}$, which determine the spacecraft field part in the total error for one magnetometer. For a given dipole field, Bdipole, the case $f=2$ can be considered as the worst case in the sense that the magnitude of the multipole fields of order $\ell \geq 2$ is appreciably above the average fields for a uniform and isotropic distribution of dipoles in the volume of the spacecraft. This is shown in the paper by Neubauer and Schatten [1972]. Specifically their analysis indicates that for an isotropic and uniform distribution of dipoles in a sphere of radius a and for the components transverse to the boom, the rms-dipole field exceeds the rms-quadrupole, rmsoctupole and the rms-16-pole fields above distances of $1.90 \mathrm{a}$, 
$1.67 \mathrm{a}$ and $1.54 \mathrm{a}$, respectively. For the usually 1 arger radial fields the corresponding numbers are $1.64 \mathrm{a}, 1.51 \mathrm{a}, 1.42 \mathrm{a}$, respectively. Since in addition large portions of the sphere of radius a approximating the MVM73-spacecraft, which is of special interest here, are empty, the transition distances would be even closer to the spacecraft. In conclusion the field models $2,4,6,8$ are rather conservative in their assumptions concerning the smallness of the multipole fields.

The models 3 and 4 give equal fields at $r_{1}=6 \mathrm{~m}$ and thereby illustrate the influence of $f=1$ and $f=2$ on the multimagnetometer errors for a boom length of $6 \mathrm{~m}$ and a given error of $2.1 \gamma$ for one magnetometer.

Table 1 also gives the magnetic field of models 1 to 8 at $r=12 \mathrm{ft}$. These values are spread around the estimate of $12.5 \mathrm{Y}$ at $12 \mathrm{ft}$ (contained in the NASA Proposal Briefing Material and assumed here to represent a magnetic field component) for Mariner-Venus-Mercury 1973. Note that models 3 and 4 are close to this estimate. The models therefore describe magnetic fields typical for spacecraft with a negligible or ineffective magnetic cleanliness program.

The results for the optimal value of the error bound $\Delta \mathrm{B}_{\max }$ for models 1 to 8 are shown in figure $1 \mathrm{a}, \mathrm{b}, \mathrm{c}, \mathrm{d}$ as a function of $r_{1}$, the boom length. Table 2 contains the results 
for models 3 and 4 for the optimal magnetometer locations and the contribution to the errors by the magnetometer inaccuracies and the factors for the calculation of the ambient field for $r_{1}=6 \mathrm{~m}$. It should be kept in mind that the maximum error $\Delta B_{\max }(\rho)$ applies to one magnetic field component. The error for one magnetometer has been calculated by using $B_{S C}\left(r_{1}\right)+B_{M}$.

\section{Discussion of Results and Conclusions}

We have solved the problem of minimizing the upper error bound for one magnetic field component for various field models and boom-lengths. The minimum is found as a tradeoff between the error due to the inaccuracy of the magnetometers and the error due to the unknown spacecraft field. The first exror is decreased by increasing the distances between the magnetometers whereas the latter error is decreased by moving the magnetometers away from the high field regions close to the spacecraft or in other words closer to each other towards the outer tip of the boom.

We first restrict ourselves to the models 1-6, in which all multipole fields have the same sign. It is seem from figures $1 \mathrm{a}, \mathrm{b}, \mathrm{c}$, that $\Delta \mathrm{B}_{\max }$ can be reduced quite appreciably by using several magnetometers. The biggest gain is achieved by going from one to two magnetometers. In the cases of the field of $2 \gamma$ at $r_{1}=6 \mathrm{~m}$ the upper bound, $\Delta B_{\max }=2.1 \gamma$ for one 
magnetometer should be compared with $\Delta B_{\max }=0.486 \gamma$ for model 3 and $0.856 \gamma$ for model 4 as shown in table 2 . The results essentially confirm those of Ness et al. [1971]. Since for smaller spacecraft fields the inaccuracy of the magnetometers represents a larger fraction of $\Delta B_{\max }$, we would expect the gains to be smaller. This is confirmed by the curves for models $1,3,5$ and models 2,4,6. Since with a given spacecraft field distribution, such as in models 1 to 6 , the individual multipole contributions fall off faster as their order increases and since the contribution caused by the inaccuracy of the magnetometers increases with the number of magnetometers, $N$, the optimal number of magnetometers decreases with increasing length of the boom $r_{1}$.

For the field models $1-6$ used in this study, which are representative for spacecraft with a restricted magnetic cleanliness programs like MVM73, the boom length at which $\Delta B_{\max }(N=1)=\Delta B_{\max }(N=2)$ lies far beyond $10 \mathrm{~m}$ even for the low field in models 1 and 2 , as figure la shows.

For magnetically clean spacecraft we find the transition distances characterised by $\Delta \mathrm{B}_{\max }(\mathrm{N}=1)=\Delta \mathrm{B}_{\max }(\mathrm{N}=2)$ very close to the spacecraft so that reasonably short booms and one triaxial magnetometer can be used. The transitions from $\Delta \mathrm{B}_{\max }(\mathrm{N}=3)<\Delta \mathrm{B}_{\max }(\mathrm{N}=2)$ to $\Delta \mathrm{B}_{\max }(\mathrm{N}=3)>\Delta \mathrm{B}_{\max }(\mathrm{N}=2)$ and the transition from $N=4$ being better than $N=3$ to the inverse relations are shown in figures $1 a, b, c$. For the 
low field model 1 and $r_{1}=4 \mathrm{~m}, N=4$ is the best choice among $N=1,2,3,4$ while for $r_{1}=6 \mathrm{~m}, N=3$ is best. For model $2 N=2$ is the best choice for all values of $r_{1}$ from $4 \mathrm{~m}$ to $10 \mathrm{~m}$.

As the spacecraft field increases, the value of $r_{1}$, at which the transition occurs, moves outward. Models 3,5,6 show the ordering $\Delta \mathrm{B}_{\max }(\mathrm{N}=4)<\Delta \mathrm{B}_{\max }(\mathrm{N}=3)<\Delta \mathrm{B}_{\max }(\mathrm{N}=2)$. Whereas in the low field models and long boom cases not very much is gained in going from 2 magnetometers to 3 or 4 magnetometers, in the strong field cases at smaller boom-lengths appreciable gains may be achieved in using 3 or 4 magnetometers. For field model 5 and $r_{1}=4 \mathrm{~m}$ a factor of 2 can be gained in going from 2 to 4 magnetometers.

Another point should be noted in comparing different values of $N$. Even if $\Delta B_{\max }(N=4) \approx \Delta B_{\max }(N=2)$ the greater effort with 4 magnetometers may be worthwile, because in $\Delta \mathrm{B}_{\max }(\mathrm{N}=4)$ the spacecraft field contribution is much smaller than in $\Delta B_{\max }(N=2)$ (see for example table 2). The error is then determined much more by the relatively well known error properties of the magnetometer than by the generally poorly known characteristics of the spacecraft field and its changes.

So far we have not considered models 7 and 8 , which are characterized by the same absolute values of the multipole 
fields in models 5 and 6 but with alternating signs. The results of these models demonstrate two logically connected points. Firstly, for given absolute values of the multipole fields, the worst case for $\Delta B_{\max }$ is the case of equal signs of all multipole fields. Secondly, in using certain field models for optimizing the multimagnetometer system, care has to be taken that the optimum is not too strongly dependent on the exact field characteristics. For example, the low values of $\Delta \mathrm{B}_{\max }(\mathrm{N}=2)$ in models 7 and 8 are caused by $\mathrm{B}_{\mathrm{RN}}=0$ at $\mathrm{r}=1.0 \mathrm{~m}$ and $\mathrm{r}=2.0 \mathrm{~m}$, respectively. This zero is given, however, as a difference of large numbers and is therefore very sensitive to changes in the spacecraft field. As the calculations of Neubauer and Schatten [1972] show, beyond approximately $3 a$, where $a$ is again the radius of the sphere enclosing the magnetic sources, the nondipolar field is dominated by the quadrupole part for most possible models. The problem just mentioned could therefore easily be resolved by imposing the further condition $r_{2} \gtrsim 2.5 a$ for the case. $N=2$ and less restrictive conditions for the innermost magnetometer in the cases $\mathrm{N}=3$ and $\mathrm{N}=4$.

Finally we note some important items which have not been considered so far: 
1. The accuracy of the magnetometer closest to the spacecraft is not critical in the overall error bound $\Delta B_{\max }$. For example, for model 3 with 4 magnetometers table 2 shows that even an error bound of $1.0 \gamma$ at $r_{4}$ would change $\Delta \mathrm{B}_{\max }$ by only $0.03 \mathrm{y}$. In addition, the optimization could also be performed with varying $B_{M, k}$. An an example we mention the final layout of the MVM73 dual magnetometer system. Since only at $r_{1}$ a flipper mechanism is used we have approximately $B_{M, 1}=0.1 \gamma$ and $B_{M, 2}=0.3 \gamma$. The actual magnetometer locations are $r_{1}=6.95 \mathrm{~m}$ and $r_{2}=4.65 \mathrm{~m}$. For the dual magnetometer system we obtain values of $\Delta \mathrm{B}_{\max }$ of $0.41 \gamma, 0.67 \gamma, 1.04 \gamma$ and $0.43 \gamma$ for models 2,4,6, 8, respectively. The corresponding values of $\Delta \mathrm{B}_{\max }$ for one magnetometer are $0.43 \gamma, 1.22 \gamma, 2.4 \gamma$ and $1.37 \gamma$ respectively. Thus for model 4 an improvement by a factor of 1.94 and for model 6 by a factor of 2.3 is achieved, although the assumption of $f=2$ can be considered to be rather conservative. These results do not change appreciably by including the fields from the solar panels. The actual magnetometer locations are optimal for $\mathrm{B}_{\mathrm{dipole}}=232 \gamma$ and $f=2$.

2. For real, finite size magnetometer-sensors, errors could also be due to gradients in the spacecraft field. The radial field component for a sensor of length $L$, which 
averages over the field along its axis as a flux-gate does, would have a gradient error of

$$
\frac{(2+l)(3+l)}{24}\left(\frac{L}{r}\right)^{2} B_{e}\left(\frac{r}{r}\right)^{l+2}
$$

due to the multipole field $B_{e}\left(\frac{r}{r}\right) l+2$. Equation 5 can easily be changed to take it into account, apart from the fact that is is very small in most practical cases.

3. Equation 5 implicitly contains the assumption that approximating the spacecraft field by the first $N-1$ multipoles is the best choice for the representation of the spacecraft field. This choice, as well as the field model employed, is dictated by the generally rather poor knowledge of the properties of the spacecraft fields. If some additional information is given in a specific case, it should be used to improve the accuracy i.e. to diminish the error bounds $\Delta B_{\max }$ along the lines sketched in section 1 between equations 7 and 10. The work started in the paper by Neubauer and Schatten [1972] can be considered as a beginning in this direction.

In conclusion we have shown that under a wide variety of assumptions about the spacecraft field, the use of two to four magnetometers can appreciably increase the accuracy of a magnetic field experiment with a magnetometer boom of reasonable 
length on a spacecraft with significant spacecraft field contributions.

ACKNOWLEDGEMENT

This work was performed under the NASA-NAS postdoctoral resident research associateship program. I greatly appreciate the hospitality of the Laboratory for Extraterrestrial Physics and discussions with Dr. R. Lepping and Dr. N.F. Ness, who also suggested this problem. 


\section{REFERENCES}

Ness, N.F., K.W. Behannon, R.P. Lepping and K.H. Schatten, Use of two magnetometers for magnetic field measurements on a spacecraft, J. of Geophys. Res. 6, 3564-3573, 1971. Ness, N.F., Magnetometers for Space Research, Space Sci. Rev., 11, 111-222, 1970.

Neubauer, F.M. and K.H. Schatten, Theoretical and observational analysis of spacecraft fields, in preparation, 1972 . 
TABLE 1 SPACECRAFT MAGNETIC FIELD MODELS

\begin{tabular}{|c|c|c|c|c|c|}
\hline Model & ${ }^{B}$ dipole & $\mathbf{f}$ & ${ }^{B}{ }_{S C}$ at $6 \mathrm{~m}$ & $\begin{array}{l}{ }^{B_{S C}} \begin{array}{l}\text { at } 12 \\
3.65 m\end{array}\end{array}$ & ft \\
\hline 1 & $100 Y$ & 1 & $0.56 \gamma$ & $2.81 \gamma$ & \\
\hline 2 & $100 \gamma$ & 2 & $0.69 Y$ & $4.29 \gamma$ & \\
\hline 3 & $360.0463 \gamma$ & 1 & $\mathbf{2} \gamma$ & $10.11 \gamma$ & \\
\hline 4 & $289.1901 \gamma$ & 2 & $2 \gamma$ & $12.40 \gamma$ & \\
\hline 5 & $550 \gamma$ & 1 & $3.06 \gamma$ & $15.45 \gamma$ & \\
\hline 6 & $550 \gamma$ & 2 & $3.80 \gamma$ & $23.59 \gamma$ & \\
\hline 7 & $550 \gamma$ & -1 & $2.18 Y$ & $8.84 Y$ & \\
\hline 8 & $550 \gamma$ & -2 & $1.92 \gamma$ & $7.62 \gamma$ & \\
\hline
\end{tabular}




\begin{tabular}{|c|c|c|c|c|c|c|c|c|c|c|}
\hline 四 & 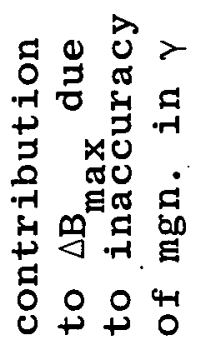 & $\begin{array}{l}N \\
0 \\
0\end{array}$ & $\begin{array}{l}\hat{N} \\
\text { N } \\
\dot{0}\end{array}$ & . & \begin{tabular}{l}
\multirow{H}{*}{} \\
$N$ \\
0
\end{tabular} & $\begin{array}{l}\text { ㅁ } \\
\dot{0}\end{array}$ & \begin{tabular}{l}
$N$ \\
\multirow{0}{*}{}
\end{tabular} & & $\stackrel{\infty}{N}$ & \\
\hline 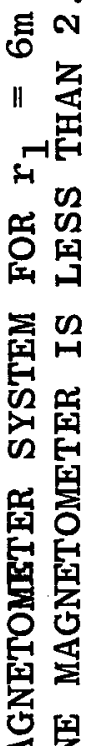 & 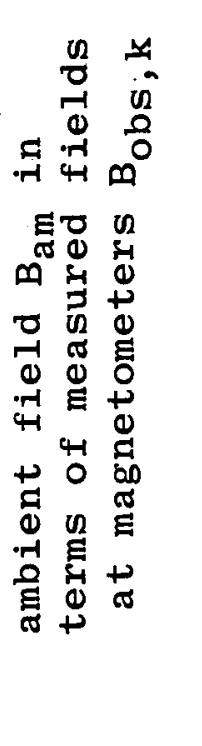 & 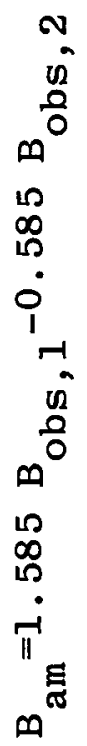 & 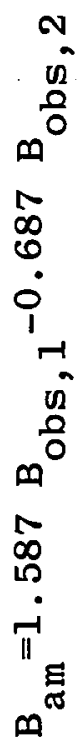 & $\begin{array}{l}m \\
\text { ñ } \\
0 \\
0 \\
0 \\
0 \\
\dot{0} \\
\dot{0} \\
+\end{array}$ & 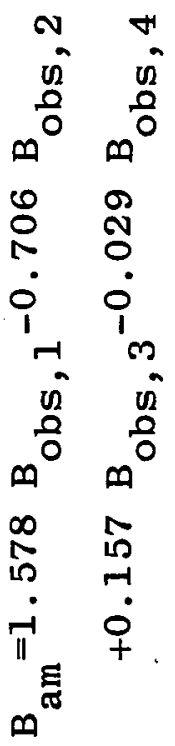 & 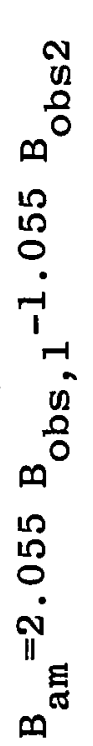 & 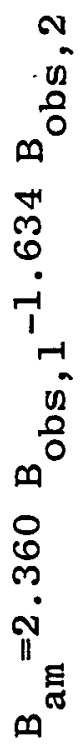 & 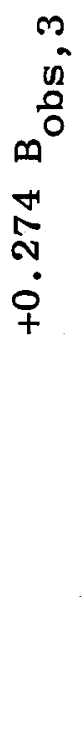 & 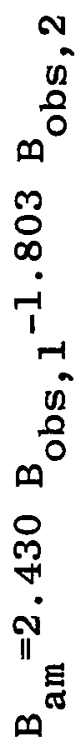 & 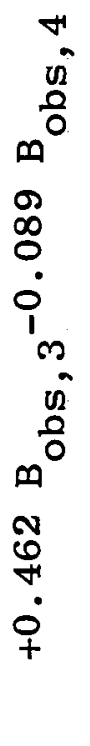 \\
\hline 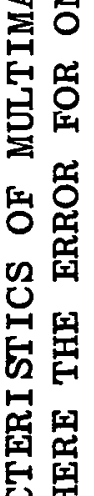 & . & 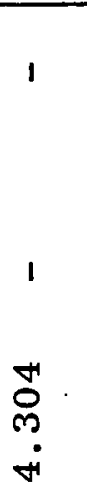 & $\begin{array}{l}0 \\
+1 \\
\dot{1} \\
\dot{0} \\
m \\
\dot{1} \\
\dot{m}\end{array}$ & & 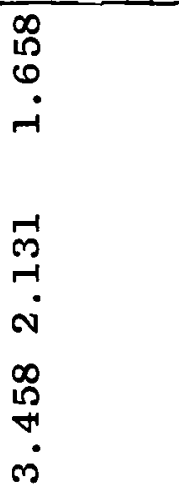 & $\begin{array}{l}1 \\
10 \\
0 \\
\infty \\
\dot{0}\end{array}$ & 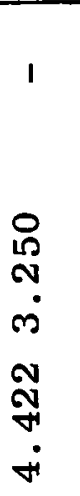 & & 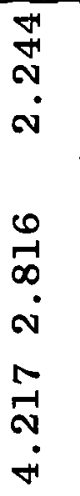 & \\
\hline 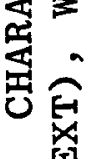 & 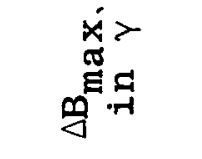 & $\begin{array}{l}\stackrel{0}{\infty} \\
\stackrel{\infty}{+} \\
\dot{0}\end{array}$ & $\begin{array}{l}\frac{\Omega}{N} \\
m \\
\dot{0}\end{array}$ & & $\begin{array}{l}10 \\
\stackrel{10}{0} \\
0\end{array}$ & $\begin{array}{l}\ddot{0} \\
\stackrel{10}{\infty} \\
\dot{0}\end{array}$ & $\begin{array}{l}\text { N } \\
\text { D } \\
\dot{0}\end{array}$ & & $\begin{array}{l}5 \\
0 \\
\infty \\
0\end{array}$ & \\
\hline 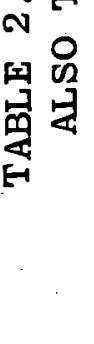 & 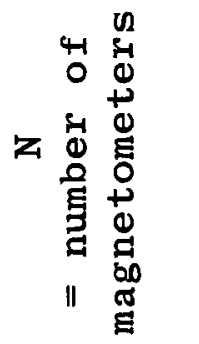 & $\boldsymbol{N}$ & $m$ & & $\forall$ & $\boldsymbol{N}$ & $m$ & & $\nabla$ & \\
\hline & $\begin{array}{l}-1 \\
0 \\
0 \\
0\end{array}$ & & $m$ & & & & $H$ & & & \\
\hline
\end{tabular}




\section{FIGURE CAPTIONS}

Fig. la-d. Upper bounds $\Delta B_{\max }$ on magnetic field errors for spacecraft field models $1-8$ as a function of boomlength. $N$ is the number of magnetometers employed. The model number refers to Table 1. 


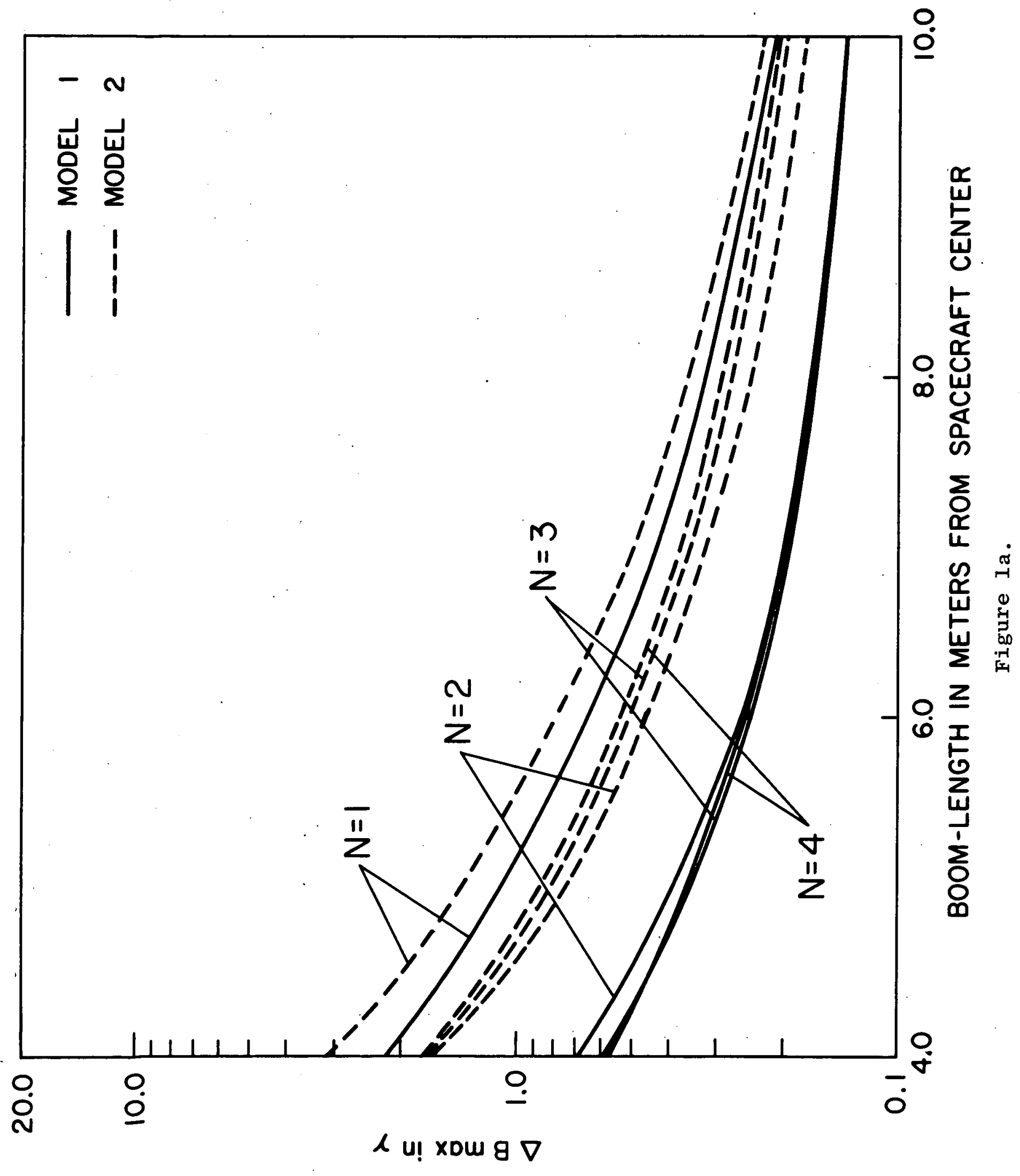




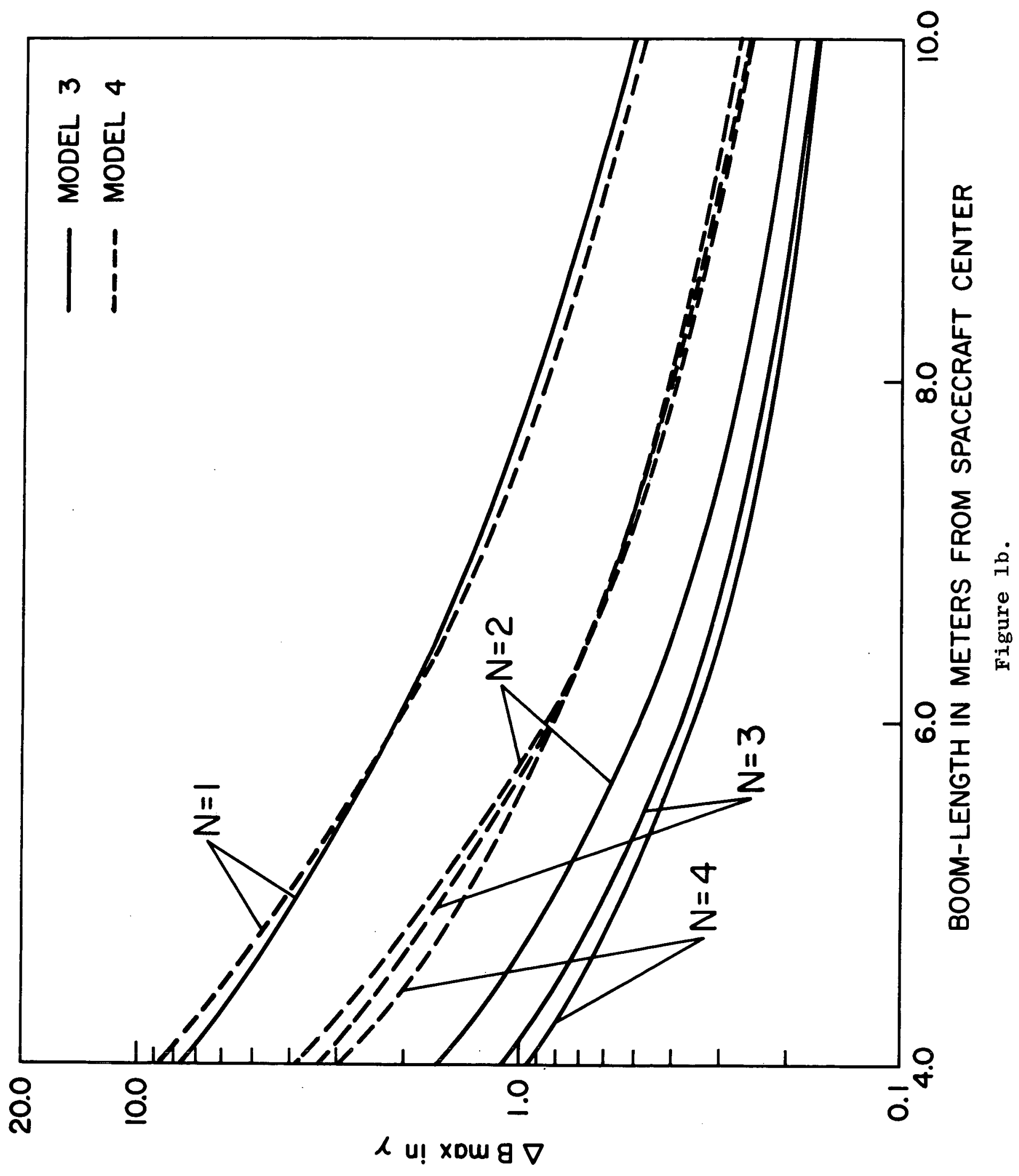




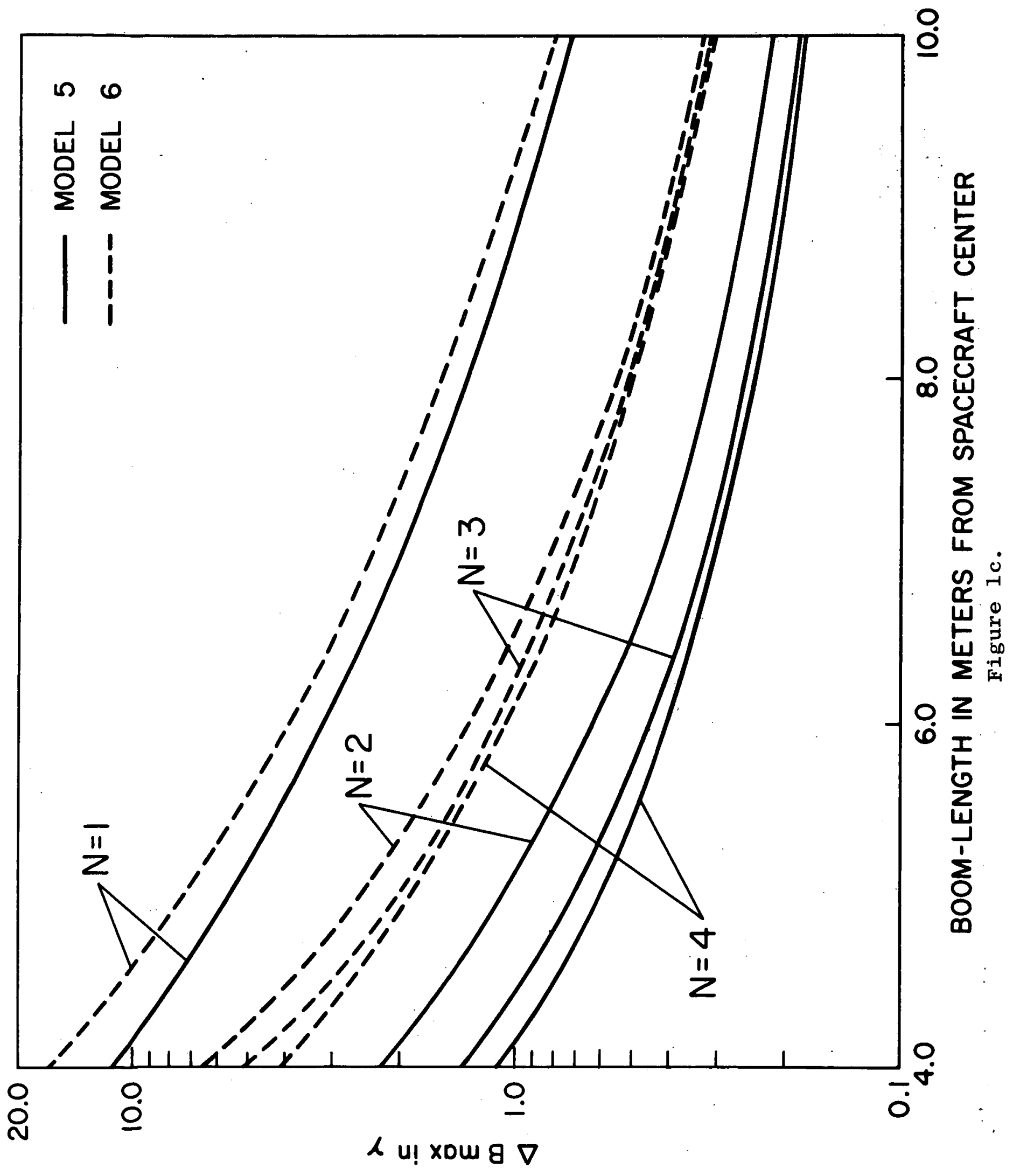




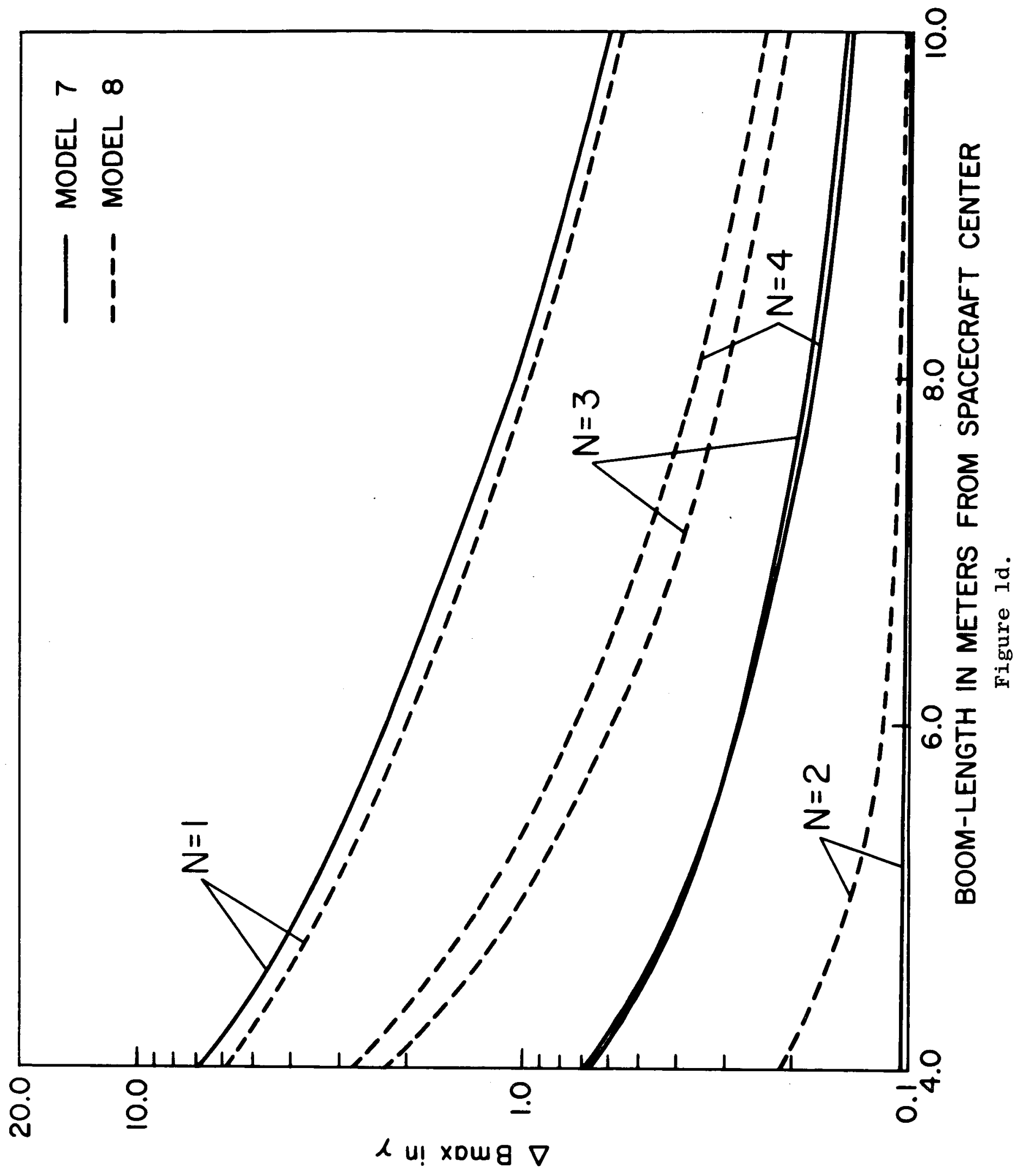

NASA-GSFC COML., Arlington, Vo. 